\title{
Sums of variables at the onset of chaos
}

\author{
Miguel Angel Fuentes ${ }^{1,2,3, a}$ and Alberto Robledo 4 \\ 1 Santa Fe Institute, 1399 Hyde Park Road, Santa Fe, 87501 New Mexico, USA \\ 2 Centro Atómico Bariloche, Instituto Balseiro and CONICET, 8400 Bariloche, Argentina \\ 3 Centro de Investigación en Complejidad Social, Facultad de Gobierno, Universidad del Desarrollo, Santiago, Chile \\ 4 Instituto de Física y Centro de Ciencias de la Complejidad, Universidad Nacional Autónoma de México, Apartado \\ Postal 20-364, México 01000 DF, Mexico
}

Received 27 September 2013

Published online (Inserted Later) - C EDP Sciences, Società Italiana di Fisica, Springer-Verlag 2014

\begin{abstract}
We explain how specific dynamical properties give rise to the limit distribution of sums of deterministic variables at the transition to chaos via the period-doubling route. We study the sums of successive positions generated by an ensemble of initial conditions uniformly distributed in the entire phase space of a unimodal map as represented by the logistic map. We find that these sums acquire their salient, multiscale, features from the repellor preimage structure that dominates the dynamics toward the attractors along the period-doubling cascade. And we explain how these properties transmit from the sums to their distribution. Specifically, we show how the stationary distribution of sums of positions at the Feigebaum point is built up from those associated with the supercycle attractors forming a hierarchical structure with multifractal and discrete scale invariance properties.
\end{abstract}

\section{Introduction}

Sums of deterministic variables, such as those generated from consecutive positions of trajectories of iterated onedimensional nonlinear maps, lead to limit distributions that reflect the periodic or chaotic character of the dynamics at work. In the former case when the Lyapunov exponent is negative, $\lambda<0$, the distribution of trajectories initiated within the attractor is trivially determined by its finite set of positions. In the latter case, when $\lambda>0$, a single chaotic trajectory (or an ensemble of them) leads to a Gaussian stationary distribution, in line with the ordinary central limit theorem, just as independent random variables do $[1,2]$. The limiting case of vanishing Lyapunov exponent $\lambda=0$ poses interesting questions about the nature of its stationary distribution: does this borderline case lead to a general type of stationary distribution, or, on the contrary, to distributions that capture the particular features of the dynamics involved? Do the absence of ergodicity and mixing preclude the appearance of broad-spectrum distributions compatible with statistical-mechanical theories? There has been speculation and discussion $[3-5]$ about whether sums of correlated deterministic variables at vanishing, or near vanishing, Lyapunov exponent give rise to a general type of non-Gaussian stationary distribution. The well-known multifractal attractor at the period-doubling transition to chaos, the Feigenbaum attractor, has proved to be a suitable model system for the exploration of this issue $[3-5]$.

\footnotetext{
a e-mail: fuentesm@santafe.edu
}

We have previously provided an answer [6,7] for the case of trajectories initiated within the Feigenbaum attractor and here we present definite conclusions for the more involved case of the dynamics towards this attractor. In the former case the support of the stationary distribution is the multifractal set that makes up the Feigenbaum attractor and its amplitude follows its multiscaling property. For the latter case we demonstrate at this time that the stationary distribution possesses an infinite-level hierarchical structure that originates from the properties of the repellor set and its preimages. This ladder organization can be more easily understood by consideration of the family of periodic attractors, conveniently, the supercycle attractors [8], along the period-doubling cascade. We bring to a close the clarification of this issue.

Specifically, we consider the logistic map $f_{\mu}(x)=$ $1-\mu x^{2},-1 \leq x \leq 1,0 \leq \mu \leq 2$, for which the control parameter value for its main period-doubling cascade accumulation point is $\mu=\mu_{\infty}=1.401155189092 \ldots$ The dynamics at and toward the Feigenbaum attractor is now known in much detail $[9,10]$ and this makes it possible to analyze the properties of sums of iterated positions that advance to this attractor with the same kind of analytic reasoning and numerical detail. A fundamental property in the analysis is the following: time evolution at $\mu_{\infty}$ from $t=0$ up to $t \rightarrow \infty$ traces the period-doubling cascade progression from $\mu=0$ up to $\mu_{\infty}[9,10]$. Beyond a close resemblance between these two developments there is asymptotic quantitative agreement. Thus, the trajectory inside the Feigenbaum attractor with initial condition $x_{0}=0$, 


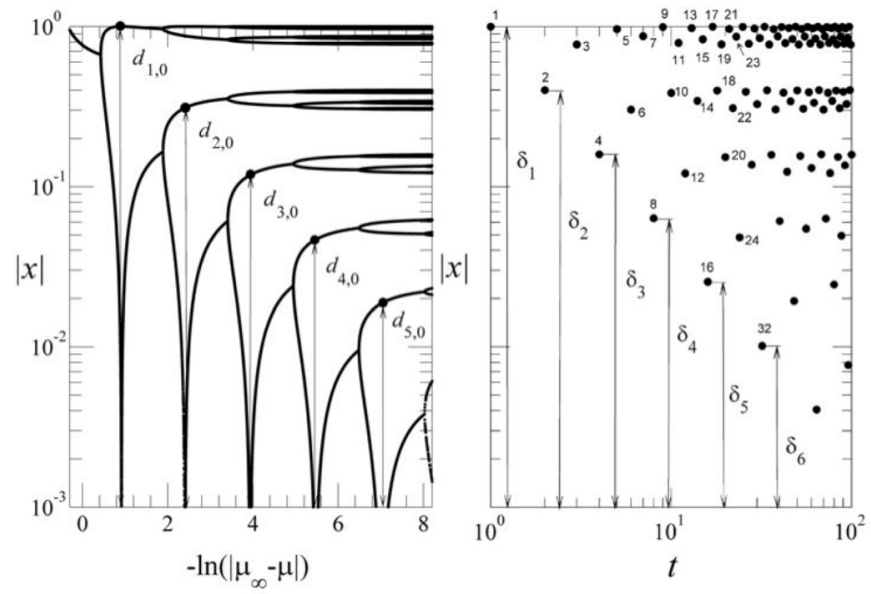

Fig. 1. Left panel: absolute value of attractor positions for the logistic map $f_{\mu}(x)$ in logarithmic scale as a function of $-\ln \left(\mu_{\infty}-\mu\right)$. Right panel: absolute value of trajectory positions for $f_{\mu}(x)$ at $\mu_{\infty}$ with initial condition $x_{0}=0$ in logarithmic scale as a function of the logarithm of time $t$, also shown by the numbers close to the circles. The arrows indicate the equivalence between the diameters $d_{n, 0}$ in the left panel, and position differences $D_{n}$ with respect to $x_{0}=0$ in the right panel.

takes positions $x_{t}$ such that the distances between nearest neighbor pairs of them reproduce the diameters $d_{n, m}$ [8] defined from the superstable, or supercycle, orbits of period $2^{n}, n=1,2,3, \ldots$, with $\bar{\mu}_{n}<\mu_{\infty}$ (see Fig. 1 ). This property has been central to obtain rigorous results for the fluctuating sensitivity to initial conditions $\xi_{t}\left(x_{0}\right)$ within the Feigenbaum attractor, as separations at chosen times $t$ of pairs of trajectories originating close to $x_{0}$ can be obtained as diameters $d_{n, m}[9]$. It has also been essential in establishing the discrete scale invariance property of the collective rate of approach of an ensemble of trajectories to this attractor where the total length of the $d_{n, m}, n$ fixed, gives the fraction of trajectories still away from the attractor at time $t=2^{n}[10]$.

Below we present results for the sums of values of iterated positions obtained from an ensemble of trajectories initially distributed uniformly along all the phase space of the logistic map. We evaluated numerically the sums for $\mu_{\infty}$ and also those for the first few supercycle values $\bar{\mu}_{n}$, $n=1,2$ and 3 . Subsequently we determined, numerically also, the stationary distributions associated with these collections of sums. To analyze the set of sums of all such positions visited by the ensemble of trajectories we record their values for all $x_{0},-1 \leq x_{0} \leq 1$, at a fixed large total final time $N$. When $\mu_{\infty}$ the set of sums is observed to have a complex structure as a function of $x_{0}$ with very rough, jagged, features that reflect the properties of the preimage arrangement of positions of trajectories on the way to the attractor, such as the long journeys taken by initial positions in the neighborhood of the multifractal repellor. The distribution of these sums is observed to have an asymmetric, exponentially-decaying, framework dressed with motifs that involve detail in many scales.

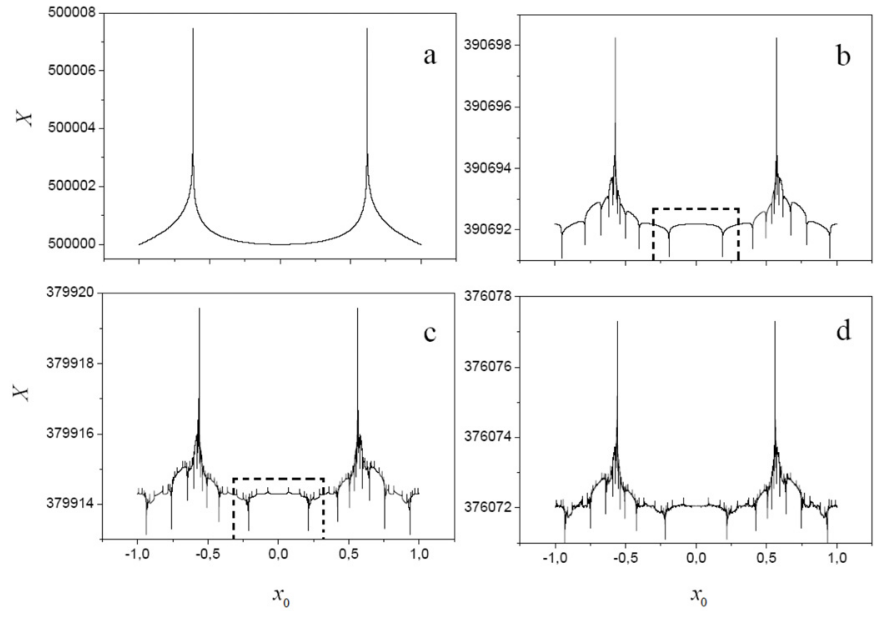

Fig. 2. Sums $X\left(x_{0}, N ; \bar{\mu}_{n}\right)$ as a function of $x_{0}, N \sim O\left(10^{6}\right)$. (a) Period 2 at $\bar{\mu}_{1}$, (b) period $2^{2}$ at $\bar{\mu}_{2}$, (c) period $2^{3}$ at $\bar{\mu}_{3}$, and (d) Feigenbaum point $2^{\infty}$ at $\mu_{\infty}$. When inverted and rescaled the area enclosed within the dashed lines in (b) and (c) become panels (a) and (b), respectively. See text for details.

\section{Sums of positions and their distributions for supercycles and their accumulation point}

We consider the sum of positions $x_{t}$ up to a final iteration time $N$ of a trajectory with initial condition $x_{0}$ and control parameter value $\mu$, i.e.

$$
X\left(x_{0}, N ; \mu\right) \equiv \sum_{t=0}^{N} x_{t} .
$$

The four panels in Figure 2 show the results for $X\left(x_{0}, N ; \mu\right)$ for all possible initial conditions $-1 \leq x_{0} \leq 1$ and $N \sim O\left(10^{6}\right)$ when the control parameter takes the values $\bar{\mu}_{1}, \bar{\mu}_{2}, \bar{\mu}_{3}$ and $\mu_{\infty}$. The plots are all symmetrical with respect to $x_{0}=0$ and exhibit two large peaks and a central valley. As it can be clearly observed, $X\left(x_{0}, N ; \bar{\mu}_{1}\right)$ for period 2 in panel (a) provides the main frame onto which motifs of alternating signs are added consecutively to produce $X\left(x_{0}, N ; \bar{\mu}_{2}\right)$ for period $2^{2}$ in panel (b), and $X\left(x_{0}, N ; \bar{\mu}_{3}\right)$ for period $2^{3}$ in panel (c). The amplitude of these motifs decreases rapidly with $n$ such that the fine features in $X\left(x_{0}, N ; \mu_{\infty}\right)$ for period $2^{\infty}$ cannot be observed at the scale of the plot in panel (d). However, zooming on a especially precise evaluation of $X\left(x_{0}, N ; \mu_{\infty}\right)$ reveals a delicate and jagged structure that becomes a multifractal when $N \rightarrow \infty$.

To appreciate better the organization of the structures of the sets of sums we plot in Figure 3 the right-hand sides with $x_{0}>0$ of the panels in Figure 2 with a logarithmic scale for the distance between the initial condition $x_{0}$ to the position $y$ for the maximum in the sums, i.e. we use the variables $w_{+}=\ln \left(x_{0}-y\right)$ and $w_{-}=\ln \left(y-x_{0}\right)$. Panel (a) of Figure 3 shows an almost linear behavior (with slope $\sigma=-1.36$ ) that indicates an exponential form around each peak of $X\left(x_{0}, N ; \bar{\mu}_{1}\right)$, a basic element preserved for $\bar{\mu}_{2}, \bar{\mu}_{3}$ and $\mu_{\infty}$ as can be observed in panels (b), (c) and (d). Next, we notice that the additional 

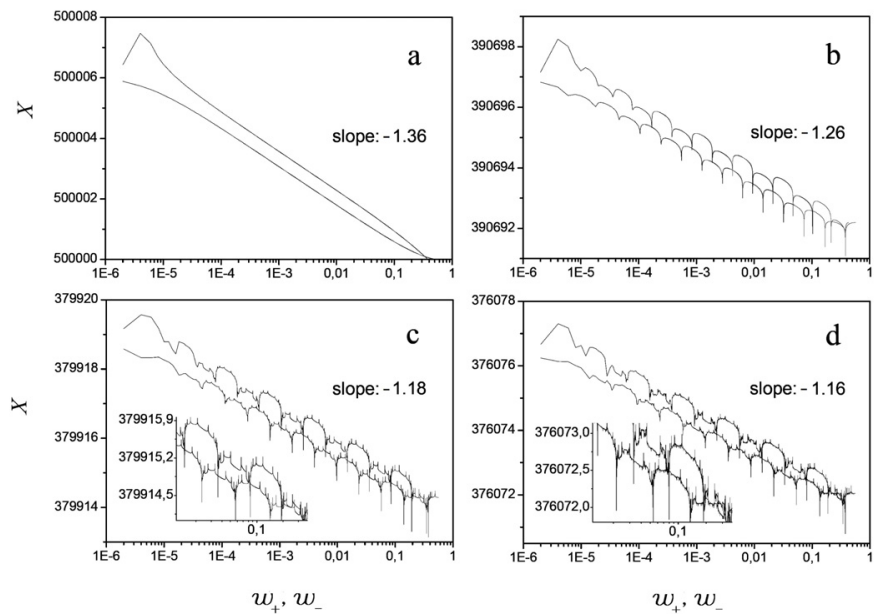

Fig. 3. Same as Figure 2 but only the sums with initial conditions $x_{0}>0$ are plotted in terms of the new variables $w_{+}=\ln \left(x_{0}-y\right)$ (upper curves) and $w_{-}=\ln \left(y-x_{0}\right)$ (lower curves). The insets show greater detail.

motifs (of smooth protrusions bounded by sharp drops) in panel (b) for $\bar{\mu}_{2}$ are spaced regularly in the logarithmic scale, a feature that will let us identify its origin below. Panel (c) for $\bar{\mu}_{3}$ shows the appearance of additional small amplitude motifs (this time small smooth valleys bounded by sharp peaks) that enhance the alternating character to the smooth motifs for $\bar{\mu}_{2}$. The scale of panel (d) for $\mu_{\infty}$ precludes the observation of fine features at the transition to chaos. The insets in panels (c) and (d) help distinguish some differences between the sums for $\bar{\mu}_{3}$ and $\mu_{\infty}$.

We proceed to evaluate the distributions associated with the sets of sums $X\left(x_{0}, N ; \mu\right)$. The four panels in Figure 4 show in semi-logarithmic scales the results for the (non-normalized) histograms that correspond to the sums in the panels with same labels in Figures 2 and 3. The essentially linear plot with slope $\Sigma_{1}=-0.76$ in panel (a) for $\bar{\mu}_{1}$ indicates an anticipated exponential decay distribution. The histogram in panel (b) for $\bar{\mu}_{2}$ shows two features in addition to that in panel (a), a repeated motif structure superposed on the line of slope $\Sigma_{1}$ and a new smooth line of slope $\Sigma_{2}=5.02$ that gives the distribution an overall asymmetrical tent shape (in the semi-logarithmic scales of Fig. 4). The histogram in panel (c) for $\bar{\mu}_{3}$ adds to the features of panel (b) both more detail to the motif structure superposed on the line of slope $\Sigma_{1}=-0.99$ and now a repeated motif structure superposed on the previously smooth line of slope $\Sigma_{2}=4.87$. These alternating finer detail appears progressively in the histograms for the distributions associated with period $2^{n}$ until in the limit $n \rightarrow \infty$ we obtain the histogram in panel (d). As before, the scale of panel (d) for $\mu_{\infty}$ precludes the observation of fine features at the transition to chaos. The insets in panels (c) and $(\mathrm{d})$ help distinguish some differences between the histograms for $\bar{\mu}_{3}$ and $\mu_{\infty}$. The stationary distribution for $\mu_{\infty}$ has an asymmetrical double exponential global shape with superposed motifs of ever-decreasing finer detail characteristic of a multifractal object. Below we explain the dynamical origin of all the features of this distribution, but
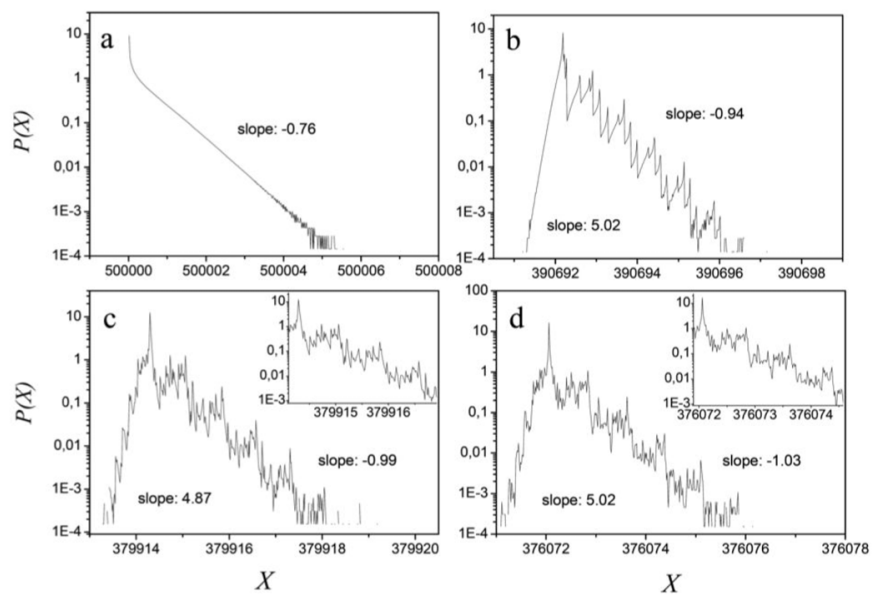

Fig. 4. Histograms obtained from the sums in Figures 2 and 3. (a) Period 2 at $\bar{\mu}_{1}$, (b) period $2^{2}$ at $\bar{\mu}_{2}$, (c) period $2^{3}$ at $\bar{\mu}_{3}$, and (d) Feigenbaum point $2^{\infty}$ at $\mu_{\infty}$. The insets show greater detail.

before we rationalize this intricate arrangement we need to refer to the dynamical properties of trajectories as they evolve towards the Feigenbaum attractor.

\section{Specifics of the dynamics towards supercycles and to their accumulation point}

For our purposes we recall some basic features of the trajectories at control parameter values $\mu=\bar{\mu}_{n}$ associated with the supercycles of periods $2^{n}, n=1,2,3, \ldots$, that are located along the bifurcation forks that form the period-doubling cascade sequence in unimodal maps (and typically illustrated by the logistic map $f_{\mu}(x)$ ). The positions (or phases) of the $2^{n}$-supercycle attractor are given by $x_{i}=f_{\bar{\mu}_{n}}^{(i)}(0), i=1,2, \ldots, 2^{n}$. Notice that infinitely many other sequences of superstable attractors appear at the period-doubling cascades within the windows of periodic attractors for values of $\mu>\mu_{\infty}$. Related to the $2^{n}$-attractor at $\mu=\bar{\mu}_{n}$ there is a $\left(2^{n}-1\right)$-repellor consisting of $2^{n}-1$ positions $y_{i}, i=1,2, \ldots, n-1$ (one for $y_{1}, 2$ for $y_{2}, 2^{2}$ for $y_{3}$, and so on). These positions are the unstable solutions, $\left|d f_{\bar{\mu}_{n}}^{\left(2^{i}\right)}(y) / d y\right|>1$, of the equations $y=f_{\bar{\mu}_{n}}^{\left(2^{i}\right)}(y), i=1,2, \ldots, n-1$. The first, $i=1$, originates at the initial period-doubling bifurcation, the next two, $i=2$, start at the 2 nd bifurcation, and so on, with the last group of $2^{i}, i=n-1$, setting out from the $(n-1)$ th bifurcation. Other families of periodic attractors along the period-doubling cascade share most of the properties of supercycles. We consider explicitly the case of a map with quadratic maximum but the results are easily extended to general nonlinearity $z>1$.

The organization of the entire set of trajectories generated by all possible initial conditions (i.e. $-1 \leq x_{0} \leq 1$ ) as they flow towards a period $2^{n}$-supercycle attractor has been studied in detail [10]. It was found that the paths taken by the full set of trajectories in their way to the attractors (or to their complementary repellors) 
are extraordinarily structured. To visualize this organization, define the preimage $x^{(k)}$ of order $k$ of position $x$ to satisfy $x=h^{(k)}\left(x^{(k)}\right)$ where $h^{(k)}(x)$ is the $k$ th composition of the map $h(x) \equiv f_{\bar{\mu}_{n}}^{\left(2^{n-1}\right)}(x)$. The preimages of the attractor of period $2^{n}, n=1,2,3, \ldots$ are distributed into different basins of attraction, one for each of the $2^{n}$ phases (positions) that compose the cycle. That is, a trajectory initiated at $x^{(k)}$ arrives after $k$ iterations at the attractor, entering first at a given position of the attractor that defines its basin ${ }^{1}$. When $n>1$ these basins are separated by fractal boundaries whose complexity increases with increasing $n$. The boundaries consist of the preimages of the corresponding repellor and their positions cluster around the $2^{n}-1$ repellor positions according to an exponential law. As $n$ increases the structure of the basin boundaries becomes more involved. Namely, the boundaries for the $2^{n}$-cycle develops new features around those of the previous $2^{n-1}$-cycle boundaries, with the outcome that a hierarchical structure arises, leading to embedded clusters of clusters of boundary positions, also crowded exponentially, and so forth. The dynamics of families of trajectories always displays a characteristically concerted order in which positions are visited, that in turn reflects the repellor preimage boundary structure of the basins of attraction. That is, each trajectory has an initial position that is identified as a preimage of a given order of an attractor (or repellor) position, and this trajectory necessarily follows the steps of other trajectories with initial conditions of lower preimage order belonging to a given chain or pathway to the attractor (or repellor). When the period $2^{n}$ of the cycle increases the dynamics becomes more involved with increasingly more complex stages that reflect the hierarchical structure of preimages (see Figs. 2 to 11 in Ref. [10] and details therein). The fractal features of the boundaries between the basins of attraction of the positions of the periodic orbits develop a structure with hierarchy, and this in turn reflects on the properties of the trajectories. The set of trajectories produces an ordered flow towards the attractor or towards the repellor that mirrors the ladder structure of the sub-basins that constitute the mentioned boundaries.

Another way by which the preimage structure described above manifests in the dynamics of trajectories moving to the supercycles of periods $2^{n}$ is via the successive formation of gaps in phase space $[-1,1]$, that in the limit $n \rightarrow \infty$ gives rise to the attractor and repellor multifractal sets. To observe explicitly this process, in reference [10] was considered an ensemble of initial conditions $x_{0}$ distributed uniformly across $[-1,1]$ and their positions were recorded at subsequent times. The set of gaps develops in time beginning with the largest one containing the $i=1$ repellor, then followed by a set of two gaps associated with the $i=2$ repellor, next a set of four gaps associated

\footnotetext{
1 We consider a trajectory "entering" the attractor at a finite number of iterations $k$ for all $x_{0}$ to be the result of a cut off in the numerical calculation, i.e. when the distance of the position $x_{k}$ to an attractor position $x_{j}$ is less than a prescribed small number [10].
}

with the $i=3$ repellor, and so forth. This process stops when the order of the gaps $i$ reaches $n-1$ (see Figs. 14 to 16 in Ref. [10] and text therein for more details).

The rate of convergence of an ensemble of trajectories to the attractor and to the repellor was measured in reference [10] by evaluating a single time-dependent quantity. A partition of phase space was made of $M_{b}$ equallysized intervals or bins and a uniform distribution of $M_{c}$ initial conditions placed along the interval $[-1,1]$, with $r=M_{c} / M_{b}$ the number $r$ of trajectories per bin. The number of bins $W_{t}$ that contain trajectories at time $t$ is shown in Figure 19 in reference [10] in logarithmic scales for the first five supercycles of periods $2^{1}$ to $2^{5}$ where the following features were observed: in all cases $W_{t}$ shows a similar initial nearly-constant plateau, and a final well-defined exponential decay to zero. In between these two features there are $n-1$ oscillations in the logarithmic scales of the figure. The duration of the overall decay grows approximately proportionally to the period $2^{n}$ of the supercycle. The dynamical mechanism at work behind the features of the decay rate $W_{t}$ is as follows: every time the period of a supercycle increases from $2^{n-1}$ to $2^{n}$ by a shift in the control parameter value from $\bar{\mu}_{n-1}$ to $\bar{\mu}_{n}$ the preimage structure advances one stage of complication in its hierarchy. Along with this, and in relation to the time evolution of the ensemble of trajectories, an additional set of $2^{n}$ smaller phase-space gaps develops and also a further oscillation takes place in the corresponding rate $W_{t}$ for finite period attractors. The rate $W_{t}$ for $\mu_{\infty}$ is shown in Figure 20 in reference [10], the time evolution tracks the period-doubling cascade progression, and every time $t$ increases from $2^{n-1}$ to $2^{n}$ the flow of trajectories undergoes equivalent passages across stages in the itinerary through the preimage ladder structure, in the development of phase-space gaps, and in logarithmic oscillations in $W_{t}$.

Concisely, each doubling of the period introduces additional modules or building blocks in the hierarchy of the preimage structure, such that the complexity of these added modules is similar to that of the total period $2^{n}$ system. As shown in reference [10], each period doubling adds also new components in the family of sequentiallyformed phase space gaps, and also increases in one unit the number of undulations in the log-periodic power-law decay displayed by the fraction $W_{t}$ of ensemble trajectories still away at a given time $t$ from the attractor (and the repellor).

\section{Explanation of the structure of the limit distribution for sums of positions at the Feigenbaum point}

We are in a position now to understand the structure of the sums in Figures 2 and 3 and their distributions in Figure 4 in terms of the dynamical properties of the trajectories that give rise to them. We start by pointing out that the peak for $x_{0}>0$ in Figure $2 \mathrm{a}$ is precisely located at the position of the $i=1$ repellor at $\bar{\mu}_{1}$, $y_{1}=-1+\sqrt{1+4 \bar{\mu}_{1}} / 2 \bar{\mu}_{1} \simeq 0.6180340 \ldots$, the solution of $y_{1}=f_{\bar{\mu}_{1}}\left(y_{1}\right)$, whereas the position of the accompanying 
peak for $x_{0}<0$ in the same panel is located at the repellor's only preimage $x_{1}^{(1)}=-y_{1}$. Actually, the sums at $x_{0}= \pm y_{1}$ are infinite when $N \rightarrow \infty$ but our calculation yields only peaks of finite height since $N<\infty$ while additionally the values of $\pm y_{1}$ are not exactly reproduced by the numerical method. Any other value of $x_{0}$ leads to a finite sum when $N \rightarrow \infty$ and to smaller values than those of the peaks when $N<\infty$. As $x_{0}$ departs gradually from $\pm y_{1}$ the values of the sums decrease monotonically generating the shape of the plot in the panel because the number of iterations required to reach the period- 2 attractor decreases accordingly ${ }^{1}$ (see Fig. 2 in Ref. [10]).This decrement in iteration numbers is exponential in $\left|y_{1}-x_{0}\right|$ and is captured by the sums as indicated in Figure 3a. The histogram in Figure 4a is an exponentially decreasing function of the sum $X$ because of the exponential shape of the latter, where the lower values of $X$ are responsible for the larger values of the histogram and vice versa. This simple shape provides the backbone of all histograms of the sums $X\left(x_{0}, N ; \mu\right)$ along the period-doubling cascade.

A drastic difference in the dynamics between period 2 and period 4 is that for the latter there is now an infinite number of preimages for the $i=1$ repellor, now located at $y_{1}=f_{\bar{\mu}_{2}}\left(y_{1}\right)$, and the same occurs to its mirror 1 st preimage located at $x_{1}^{(1)}=-y_{1}$. The basins of attraction of the $2^{2}$-attractor positions have now fractal boundaries. The preimages of either $\pm y_{1}$ on these boundaries cluster exponentially towards $\pm y_{1}$ (as can be seen in Figs. 4 and 5 in Ref. [10]). The two positions of the $i=2$ repellor, the solutions of $y_{2}=f_{\bar{\mu}_{2}}^{(2)}\left(y_{2}\right)$, have each only single preimages located symmetrically at $x_{2}^{(1)}=-y_{2}$. The (infinite number of) singular cusp shapes that point downwards in Figures $2 \mathrm{~b}$ and $3 \mathrm{~b}$ also cluster exponentially towards the positions $\pm y_{1}$ and appear precisely located on the preimages of either $\pm y_{1}$. The sums with $x_{0}$ located on the preimages of $\pm y_{1}$ are local minima for the following reasons: first, the trajectories initiated in the close vicinity of any such locations flow towards the $i=2$ repellor $y_{2}$ (two positions $y_{2}^{(1)}$ and $\left.y_{2}^{(2)}\right)$ or to its preimage $-y_{2}$ (two positions $-y_{2}^{(1)}$ and $\left.-y_{2}^{(2)}\right)$ after which they perform a long quasi period-2 cycle around the two positions $y_{2}^{(1)}$ and $y_{2}^{(2)}$ before falling into the period- 4 attractor (see, for example, Fig. 12 in Ref. [10]). Second, $y_{2}^{(1)}>0$ and $y_{2}^{(2)}<0$. Therefore the flow out of any of the preimages of $\pm y_{1}$ involves a large amount of cancellations in the corresponding sums, more than for any other initial $x_{0}$, and this leads to the sharp dips in Figures $2 \mathrm{~b}$ and $3 \mathrm{~b}$. The exponential spacing of the motifs just described in the plots for $X\left(x_{0}, N ; \bar{\mu}_{2}\right)$ generates the regular seesaw shape in semi-logarithmic scales of the corresponding histogram in Figure 4b. The fluctuating pattern consists of repetition of four oscillations that are associated with the sinks just described in the sums since there are four sets of them, two of which are shown in Figure $3 \mathrm{~b}$ and the other two are their mirror images for $x_{0}<0$. The smooth line of positive slope that creates the tent shape of the histogram originates from the structures of $X\left(x_{0}, N ; \bar{\mu}_{2}\right)$ around the 2 nd generation repellor positions and their preimages at $\pm y_{2}^{(1)}$ and $\pm y_{2}^{(2)}$ that have a scaled, inverted, shape of the total sum $X\left(x_{0}, N ; \bar{\mu}_{1}\right)$ in Figure 2a (see the central region enclosed by dashed lines in Fig. 2b).

The passage from period 4 to period 8 introduces another level of complication to the dynamics towards the attractor. Where there was before an infinite number of preimages for the $i=1$ repellor clustering exponentially around it, there is now an infinite number of such clusters, formed by preimages clustering exponentially around the period 4 preimages with shifted locations from $\bar{\mu}_{2}$ to $\bar{\mu}_{3}$. The clusters themselves cluster around the $i=1$ repellor, now located at $y_{1}=f_{\bar{\mu}_{3}}\left(y_{1}\right)$. And likewise for its 1 st preimage $-y_{1}$. Where there were before only two preimages to the two positions of the $i=2$ repellor, there is now an infinite number of preimages clustering exponentially around them, now located at the solutions of $y_{2}=f_{\bar{\mu}_{3}}^{(2)}\left(y_{2}\right)$. And likewise for its two 1 st preimages $-y_{2}$. Additionally, there are now four positions of the $i=3$ repellor, the solutions of $y_{3}=f_{\bar{\mu}_{3}}^{\left(2^{2}\right)}\left(y_{3}\right)$, that have each only one preimage located symmetrically at $x_{3}^{(1)}=-y_{3}$ (for more details see Figs. 6 and 7 in Ref. [10]). We observe now in Figures $2 \mathrm{c}$ and $3 \mathrm{c}$ the appearance of new sets of spikes in $X\left(x_{0}, N ; \bar{\mu}_{3}\right)$, and the spikes within each set cluster exponentially around each of the sharp dips that appeared 1st in Figures $2 \mathrm{~b}$ and $3 \mathrm{~b}$. These new sets of spikes correspond to the clusters of repellor preimages that, like the clusters themselves, cluster exponentially around the $i=1$ repellor and its 1st preimage; this because, as we have seen, the sharp dips do likewise. The exponential spacing of the clusters of preimages and of the exponential clustering of the preimages themselves within clusters just described in the plots for $X\left(x_{0}, N ; \bar{\mu}_{3}\right)$ generate a more intricate seesaw shape in semi-logarithmic scales of the corresponding histogram in Figure 4c. The branch of positive slope that creates the tent shape of the histogram originates from the structures of $X\left(x_{0}, N ; \bar{\mu}_{3}\right)$ around the 3rd generation repellor positions and their preimages at $\pm y_{3}^{(1)}$ and $\pm y_{3}^{(2)}$, $\pm y_{3}^{(3)}$ and $\pm y_{3}^{(4)}$, that have a scaled, inverted, shape of the total sum $X\left(x_{0}, N ; \bar{\mu}_{2}\right)$ in Figure $2 \mathrm{~b}$ (see the central region enclosed by dashed lines in Fig. 2c). For this reason we observe that the seesaw pattern complexity of the positive slope branch of the tent-like histogram for each $2^{n}$-supercycle, is equivalent to the negative slope branch of the previous $2^{n-1}$-supercycle.

The alternation of upward and downward cusps in the sums $X\left(x_{0}, N ; \bar{\mu}_{n}\right), n=1,2,3, \ldots$, is due to the fact that the repellor positions $y_{n-1}$ and 1st preimages $-y_{n-1}$ fall within the bands of attractor positions shown in Figure 1b. In this figure (from top to bottom) the 1st, 3rd, 5th, etc., bands contain all the positive positions, $x>0$, while the $2 \mathrm{nd}, 4 \mathrm{th}, 6 \mathrm{th}$, etc., bands have all the negative positions, $x<0$. As partly mentioned, trajectories at $\bar{\mu}_{n}$ initiated close to $\pm y_{1}$ flow towards the $2^{2}$ positions $\pm y_{2}$, to undergo a long quasi period-2 cycle around the two repellor positions $y_{2}^{(1)}$ and $y_{2}^{(2)}$. Then they proceed to flow towards the $2^{2}$ positions $y_{3}$, to undergo a longer quasi 
period- $2^{2}$ cycle around the $2^{2}$ repellor positions $y_{3}^{(1)}, y_{3}^{(2)}$, $y_{3}^{(3)}$, and $y_{3}^{(4)}$. This process continues until the $y_{n-1}$ repellor positions are reached and finally fall into the period- $2^{n}$ attractor (see Fig. 12 in Ref. [10]). These generic flow out of any of the preimages of $\pm y_{1}$ (or out of any of the preimages of $\left.\pm y_{i}, i=2,3, \ldots n-1\right)$ involves a large amount of cancellations in the corresponding sums (more than for any other $x_{0}$ ), and leads to sharp cusps. The smallest cusps point upwards or downwards when $n$ is odd or even, respectively, as this condition places the $y_{n-1}$ repellor positions within an odd or even band in Figure $1 \mathrm{~b}$.

Bearing in mind the basic property that trajectories at $\mu_{\infty}$ from $t=0$ up to $t \rightarrow \infty$ trace the period-doubling cascade progression from $\mu=0$ up to $\mu_{\infty}$, it is clear now how to decode the structure of the sums in Figures $2 \mathrm{~d}$ and $3 \mathrm{~d}$ and that of their histogram in Figure 4d. The arrangement of the multiscale families of cusps of $X\left(x_{0}, N ; \mu_{\infty}\right)$ is the manifestation of the consecutive formation of phase space gaps in an initially uniform distribution of positions $x_{0}$, and the logarithmic oscillations at times $t^{k}$, $k=1,2,3, \ldots$, of the rate of convergence of trajectories $W_{t}$ to the Feigenbaum attractor. The mean time for the opening a gaps of the same order is $t^{k}, k=1,2,3, \ldots$, and the signs and amplitudes of the cusps are the testimonies stamped in the sums of the main passages out of the laberynths formed by the preimages of the repellor, the transits of trajectories from one level of the hierarchy to the next. Visual representation of the stationary distribution $P\left(X, N \rightarrow \infty ; \mu_{\infty}\right)$ associated with the sums $X\left(x_{0}, N ; \mu_{\infty}\right)$ is shown by the histogram in Figure $4 \mathrm{~d}$. It has an asymmetrical double exponential backbone onto which multiscale patterns are attached that originate from the aforementioned sets of cusps in the sums. The negative slope (in semi-logarithmic scales) of the backbone originates from the main $k=1$ repellor core structure shown for all supercycles while the steeper positive slope originated form the replica structures of the former that originate from the $k=2$ repellor structures.

\section{Summary and discussion}

We have shown that the sums of iterated positions of an ensemble of trajectories moving toward the Feigenbaum attractor have a multiscale, hierarchical, structure that exhibits the preimage organization of its corresponding repellor. The building blocks of the hierarchy were identified by looking at the analogous sets of sums obtained from the dynamics of approach to the simpler supercycle attractors along the period-doubling cascade. Figures $2 \mathrm{a}-2 \mathrm{c}$, as well as Figures $3 \mathrm{a}-3 \mathrm{c}$, demonstrate clearly how the structure shown in Figures 2d and 3d for the sums at the perioddoubling accumulation point develops.

This basic property suggests a narrow degree of universality for the sums of deterministic variables at the transitions to chaos, limited to the universality class of the route to chaos under consideration. Namely, the sums of positions of memory-retaining trajectories evolving under a vanishing Lyapunov exponent appear to preserve the particular features of the multifractal critical attractor and repellor under examination. Thus we expect that varying the degree of nonlinearity of a unimodal map would affect the scaling properties of sums or time averages of trajectory positions at the period-doubling transition to chaos, or alternatively, that the consideration of a different route to chaos, such as any of the quasiperiodic routes, would lead to a different structure of comparable time averages.

As described above, the spiked functional dependence of the sum $X\left(x_{0}, N ; \mu_{\infty}\right)$ on $x_{0}$ observable in Figures $2 \mathrm{~d}$ and $3 \mathrm{~d}$ follows the characteristic hierarchical preimage structure, with exponential clustering, around the positions of the major and other high-ranking elements of the repellor and their first preimages [10]. This feature suggests that large sums of positions are dominated by long journeys toward the attractor that are particular to the attractor under consideration at the transition to chaos.

The distributions associated with large sums of positions acquire also a multiscale, hierarchical, structure, as the nature of $X\left(x_{0}, N \mu_{\infty}\right)$ is transferred, although in a different setting shown in the histogram in Figure $4 \mathrm{~d}$, to their distribution $P\left(X, N ; \mu_{\infty}\right)$. Again, the building blocks of the hierarchy in the distribution are revealed by determination of the analogous distributions of the sums $X\left(x_{0}, N ; \bar{\mu}_{n}\right)$ associated with the supercycle attractors. Figures $4 \mathrm{a}-4 \mathrm{c}$ show plainly how the structure in Figure $4 \mathrm{~d}$ for the histogram at the period-doubling accumulation point builds up stage by stage. Parallel to the period-doubling cascade that contains the supercycles at $\bar{\mu}_{n}<\mu_{\infty}$, there is a chaotic band-splitting cascade at control parameter values $\widehat{\mu}_{n}>\mu_{\infty}$ that converge also to $\mu_{\infty}$ [8]. The set of chaotic-band attractors at $\widehat{\mu}_{n}$ (formed by $2^{n}$ bands) cannot be used as we have done here for the supercycles at $\bar{\mu}_{n}$ to determine the stationary distribution $P\left(X, N ; \mu_{\infty}\right)$ because the width of the bands present at $x_{0}$ is gradually increased as the number of terms in the sums increases and they merge covering all the interval $[-1,1]$. Because the intraband motion is chaotic the sums become equivalent to sums of independent random variables and a stationary Gaussian distribution is obtained when $N \rightarrow \infty[6,7]$.

The results presented here are valid for a finite but large number of summands $N \sim O\left(10^{6}\right)$, i.e. long trajectories, and the numerical results are dependent also on the fine but finite subdivision of the phase space interval $[-1,1]$, with a number of bins $M \sim O\left(10^{6}\right)$ each with a trajectory initial condition $x_{0}$. Moving towards the limit $M \rightarrow \infty$ places initial conditions $x_{0}$ closer to the repellor or their preimage positions enlarging the heights of the spikes in the sums and this requires increasing $N \rightarrow \infty$ to observe them in Figures 2 and 3. On the other hand dumping a number of initial terms in the sum $X\left(x_{0}, N ; \mu_{\infty}\right)$ has the effect of clipping the largest spikes in it and leading to a smoother distribution. However $X\left(x_{0}, N ; \mu_{\infty}\right)$ and its distribution are never going to be even, differentiable functions, for any number of discarded terms. Moreover, the progress toward a limit distribution is not supposed to involve selected removal of terms. 
In the ordinary CLT the sum of independent random variables is equivalent to the convolution of distributions and as this operation is repeatedly applied one obtains a Gaussian distribution independently of their initial (finite variance) distribution. The summation process of trajectory positions we have studied here can be seen as the gradual transformation of an initial distribution, uniform in our case, into a limiting form by the dynamical action of the attractor and repellor under vanishing Lyapunov exponent. We have seen that the particular features of such an attractor/repellor pair are imprinted in the resulting stationary distribution. Ad hoc attractor/repellor pairs could be used to construct specific limiting distributions.

MAF thanks CONICYT (Chile): Anillo en Complejidad Social and Proyecto Interfacultades, Universidad del Desarrollo. AR acknowledges support from DGAPA-UNAM-IN100311 and CONACyT-CB-2011-167978 (Mexican Agencies).

\section{References}

1. N.G. van Kampen, Stochastic Processes in Physics and Chemistry (North-Holland Pub., Amsterdam, 1981)

2. A.Ya. Khinchin, Mathematical Foundations of Statistical Mechanics (Dover Pub., New York, 1949)

3. U. Tirnakli, C. Beck, C. Tsallis, Phys. Rev. E 75, 040106(R) (2007)

4. U. Tirnakli, C. Beck, C. Tsallis, Phys. Rev. E 79, 056209 (2009)

5. P. Grassberger, Phys. Rev. E 79, 057201 (2009)

6. M.A. Fuentes, A. Robledo, J. Stat. Mech. 2010, P01001 (2010)

7. M.A. Fuentes, A. Robledo, J. Phys.: Conf. Ser. 201, 012002 (2010)

8. H.G. Schuster, Deterministic Chaos. An Introduction, 2nd revised edn. (VCH Publishers, Weinheim, 1988)

9. E. Mayoral, A. Robledo, Phys. Rev. E 72, 026209 (2005)

10. A. Robledo, L.G. Moyano, Phys. Rev. E 77, 036213 (2008) 